\title{
Survival Survey of Lactobacillus acidophilus In Additional Probiotic Bread
}

\author{
Truong Duc Thang ${ }^{1, a}$, Le Thi Hanh Quyen ${ }^{1, b}$, Hoang Thi Thuy Hang1,c, Nguyen Thien Luan ${ }^{1, d}$, \\ Dang Thi KimThuy ${ }^{2, e}$, Lieu My Dong 1, f,*
}

${ }^{I}$ Faculty of Food Technology, Ho Chi Minh city University of Food Industry, Vietnam

${ }^{2}$ Institute of Tropical Biology, Ho Chi Minh city, Vietnam

*Corresponding author

\section{A R T I C L E I N F O}

Research Article

Received : 03/10/2018

Accepted : 19/02/2019

\section{Keywords:}

Alginate

Lactobacillus acidophilus

Mmaltodextrin

Xanthan gum

Probiotic bread
A B S T R A C T

Bread is a popular food in the world because of its variety and convenience. Currently, studies on the adding probiotics to bread are limited due to the adverse effects of processing, such as baking temperature, aerobic environment to the probiotic bacteria. The objective of this study was to produce probiotic cream bread, in which Lactobacillus acidophilus was microencapsulated with Alginate $2 \%$ (A); Alginate 2\% + maltodextrin 1\% (AM); Alginate 2\% + xanthan gum $0.1 \%(\mathrm{AX})$; and Alginate $2 \%+$ maltodextrin $1 \%+$ xanthan gum $0.1 \%$ (AMX). Microcapsules were added to the kernel, conducting encapsulation yield investigations, survival in baking, preservation of bread, and in simulated gastric fluid and simulated intestinal fluid conditions after 8 days of storage. The results showed that the addition of xanthan gum enhanced the encapsulation yield, it reached $92.9 \%$ and 92.37\% in AMX and AX samples, respectively. The viability of L. acidophilus during baking was decreased by 3.64 and $3.75 \mathrm{Log}$ (CFU/bread) in AMX and AM samples, compared to A and AX which were decreased by 4.75 and $4.44 \mathrm{Log}$ (CFU/ bread). In SGF (Simulated Gastric Fluid) and SIF (Simulated Intestinal Fluid) conditions, the AMX microcapsules provide the best probiotic protection among the four tested carriers. The combination of xanthan gum and maltodextrin in alginate matrix, eventually leading to having dual efficiency: First, xanthan gum would act as buffers that reduce acid activity; Second, maltodextrin acting as a protective agent of $L$. acidophilus against high temperature as well as potential prebiotic that improve the viability of probiotic. truongducthang1996@gmail.com
hoangthuyhanggl@gmail.com dtkthuy@gmail.com

\section{(iD) https://orcid.org/0000-0002-2015-3122 $\left.\right|^{\mathbf{b}}$ hanhquyen999.ql@gmail.com (1)https://orcid.org/0000-0002-9815-9786 d thienluannguyen1997@gmail.com \\ iD https://orcid.org/0000-0002-4212-6942 $\left.\right|^{\mathrm{P}}$ lieudong289@gmail.com}

(iD) https://orcid.org/0000-0002-7547-6076 (iD) https://orcid.org/0000-0002-0774-127X (iD) https://orcid.org/0000-0003-2836-5290

(c) (1) (8) This work is licensed under Creative Commons Attribution 4.0 International License

\section{Introduction}

FAO/WHO defines probiotics as "Live microorganisms (bacteria or yeasts), which when ingested or locally applied in sufficient numbers confer one or more specified demonstrated health benefits for the host" (Hill et al., 2014). They contribute significantly to improve human health and safety such as improving digestion and nutrition, preventing the development of harmful organisms, preventing diarrhea, preventing or reducing food allergies, helping to create antibodies to improve immunity, treatment of infectious diseases (Hatakka et al., 2001). However, to ensure the benefits of probiotics, the number of probiotic bacteria must exceed $10^{6} \mathrm{CFU} / \mathrm{mL}$ at products' consumption time (Agrawal, 2005).

Bread is the main food in many countries because it is a source of carbohydrates, proteins, vitamins and minerals. The development of bread products including both providing nutrition and bringing health benefits which are main concerns nowadays. The addition of probiotic to bread is considered an effective way to introduce these beneficial effects into human meals (Kailasapathy and
Chin, 2000). However, the processing process becomes a major barrier of adding probiotics. The effects of probiotics bacteria on food depend on the type of food, the dosage used, and the existence of air (Homayouni et al., 2007). Their viability must be ensured throughout the life of the product and in the digestion system (Kailasapathy and Chin, 2000).

Microencapsulation technology with calcium alginate proves the survival potential of probiotic. Alginate is a food additive, non-toxic, and it bonds with calcium chloride to fix and protect fundamental cells with low cost (Sultana et al., 2000). In contrast, the use of alginate is restricted due to instability in low $\mathrm{pH}$ conditions. The combination of alginate with prebiotic such as maltodextrin improves the viability of probiotics and microcapsules (Ding and Shah, 2007). Recent studies have also reported that xanthan gum combined with alginate improves probiotics protection in simulated gastric fluid (SGF) and simulated intestinal fluid (SIF) (Chen et al., 2017). However, the combination of these three compounds to protect probiotic bacteria during 
bread production and preservation has not been published in previous studies. This study carried out experiments to evaluate the microbial performance, viability of microbial probiotics by carriers Alginate $2 \%$, Alginate $2 \%+$ Maltodextrin 1\%, Alginate 2\% + Xanthan gum $0.1 \%$, Alginate 2\% + Maltodextrin $1 \%+$ Xanthan gum $0.1 \%$ and supplementation in bread with adverse factors which are baking temperature, storage, and survival of L. acidophilus in vitro and in gastric and bile salts. From these data, the best type of carrier is identified to supplement the probiotic microcapsules with bread.

\section{Materials and methods}

\section{Microorganisms and Micro-Encapsulation}

Lactobacillus acidophilus ATCC 4356 was obtained from stain collection of Faculty of food technology of the Ho Chi Minh University of food industry. Lactobacillus acidophilus was harvested from $100 \mathrm{ml}$ of a 22-h culture (late log phase) by centrifugation at $5000 \mathrm{rpm}$. The cells were then washed twice and resuspended in $10 \mathrm{ml}$ of sterile saline and used in the microencapsulation process.

\section{Prepare Microcapsule Preparation and Test Encapsulation Yield}

Microcapsule preparation was made by an emulsifying method which was described in the study by Sultana et al., (2000) with slight modification. Briefly, 20ml of alginate $2 \%$ (A); alginate $2 \%$ + maltodextrin $1 \%$ (AM); alginate $2 \%$ + xanthan gum $0.1 \%(\mathrm{AX})$ and alginate $2 \%+$ Maltodextrin $1 \%+0.1 \%$ xanthan gum (AMX) were mixed with $5 \mathrm{ml}$ of L. acidophilus. The mixture was stirred by a magnetic stirrer and dispersed for 15 minutes in $100 \mathrm{ml}$ of oil containing tween $801.5 \%(\mathrm{v} / \mathrm{v})$ at a speed of 900 rotation per minutes. After 5 minutes, added slowly $150 \mathrm{~mL}$ of $\mathrm{CaCl}_{2}$ 0.05 $\mathrm{M}$ solution to break the emulsion. Collect postemulsion and preserve at $4^{\circ} \mathrm{C}$ to conduct experiments. Examined encapsulation yield in the microcapsule preparation and calculated according to the following formula:

$$
\text { Encapsulation yield }(\%)=\frac{\sum \log C F U_{\text {after }}}{\sum \log C F U_{\text {before }}} \times 100 \%
$$

Prepare Bread, Check the Survival of Bacteria During Baking and Storage

The kernel of bread was prepared in the following main proportions: Wheat flour $(10 \% \mathrm{w} / \mathrm{v})$, fresh milk $(40 \% \mathrm{v} / \mathrm{v})$, egg yolk $(10 \% \mathrm{w} / \mathrm{v})$, sugar $(22 \% \mathrm{w} / \mathrm{v})$ with the proper ratio, then, cook it and leave it cool. Then, mix microcapsule preparation with the ratio of $4: 1$, and divide it into 5 grams pieces and storage at $-18^{\circ} \mathrm{C}$ for $20 \mathrm{~min}$. Then add to the prepared cake. The bread crust was prepared in the following main proportions: wheat flour $(45 \% \mathrm{w} / \mathrm{v})$, yeast $(5 \% \mathrm{w} / \mathrm{v})$, sugar $(6 \% \mathrm{w} / \mathrm{v})$, milk (20\% v/v), eggs (5\% w/v), butter $(20 \% \mathrm{w} / \mathrm{v}) \ldots$ mixed with water in appropriate proportion, divide into smaller pieces, each piece was 50 grams. After, incubated them at $32^{\circ} \mathrm{C}$ within 1 hour 30 minutes. Conduct baking at $185^{\circ} \mathrm{C}$ for 13 minutes. Bread was cooled down and stored at $4^{\circ} \mathrm{C}$. Samples contain free cells as samples to confront. The viability of L. acidophilus was tested according to the following formula:

$$
\text { Survival }(\%)=\frac{\sum \log C F U_{\text {after baking }}}{\sum \log C F U_{\text {before baking }}} \times 100 \%
$$

Bread samples were preserved at $4^{\circ} \mathrm{C}$ and tested for the viability of $L$. acidophilus were examined indirectly at time intervals of $0,2,4,6,8$ days of storage.

Effects of Simulated Gastric Fluid (SGF) And Intestinal Fluid (SIF) On L. Acidophilus After 8 Days of Storage

Five grams of bread kernel after 8 days of storage were incubated in $45 \mathrm{ml}$ of SGF solution $(9 \mathrm{~g} / 1 \mathrm{NaCl}+3 \mathrm{~g} / \mathrm{l}$ pepsin (Himedia) adjusted to $\mathrm{pH} 2.5$ with $5 \mathrm{~N} \mathrm{HCl}$ ), at $37^{\circ} \mathrm{C}$ for 120 minutes. After incubation in SGF, the samples were collected and transferred to $45 \mathrm{ml}$ of SIF (simulated intestinal fluid) $(0.85 \% \mathrm{NaCl}, 0.3 \%$ bile salts, $\mathrm{pH} 6.5)$ incubated at $37^{\circ} \mathrm{C}$ for the next 3 hours. The viability of $L$. acidophilus from microspheres was immediately assayed by plating on MRS media.

\section{Statistical Analysis}

All the experiments were repeated three times, and the results were presented as average \pm SD. Analysis of variance ANOVA $(\mathrm{P}<0.05)$ was used for the mean of average comparison. All statistical calculations are done using STATGRAPHICS software

\section{Results and Discussion}

\section{Encapsulation Yield of Carrier Matrix with L.} acidophilus

Encapsulation yield of L. acidophilus of carrier matrix was demonstrated in Fig. 1. The results indicated that the used carrier matrix brought effectiveness more (80\%). Carriers that had the highest encapsulation yield was AMX which has the same result of carrier AX $(\mathrm{P}>0.05)$. The results were approximately $92.9 \%$ and $92.37 \%$. The lower efficiency carriers were AM and A which was $86.7 \%$ and $86.32 \%$.

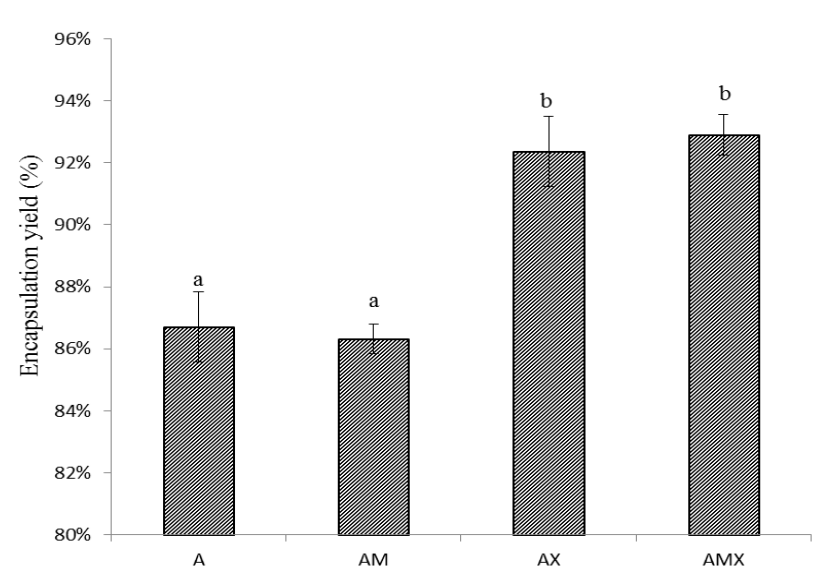

Fig 1. Encapsulation yield of carriers (A: alginate 2\%; AM: alginate $2 \%+$ maltodextrin $1 \%$; AX: alginate $2 \%+$ xanthan gum $1 \%$; AMX: alginate $2 \%+$ maltodextrin $1 \%+$ xanthan gum $0,1 \%$ ). ${ }^{a-b}$ represents for the significant difference $(\mathrm{P}<0.05)$ in each experience. 
Encapsulation yield of probiotics is necessary for considering in adding probiotic into bread products. The higher the yield, the higher the number of microorganisms in products, resulting in reducing the number of microcapsules needed to be added to the bread kernel, thereby reducing the sensory value of bread products with probiotic. Trabelsi et al. (2014) conducted a test on the encapsulation yield of L. plantarum with alginate at different concentrations $(1 \%, 2 \%$ and $3 \% \mathrm{w} / \mathrm{v})$ showed that $2 \%$ alginate concentration gave the best results (Trabelsi et al., 2014). The study by Fareez et al. (2015) showed that microparticles were emulsified with alginate carriers; Alginate - Xanthan gum; Alginate - Xanthan gum Chitosan; Alginate - Chitosan, the result states that the encapsulation yield has the existence of alginate and xanthan gum achieve more than 90\% (Fareez et al., 2015).

In the present study showed that the different carrier components significantly affected the encapsulation yield of the L. acidophilus, in which the complex microcapsule containing the xanthan gum component (AX and AMX) gave encapsulation yield higher than others in the same research $(\mathrm{P}<0,05)$ (Fig 1). This suggests that xanthan gum contributes significantly to the encapsulation yield of the carrier that adds xanthan gum.

Survival Ability of L. acidophilus During Baking Process and Storage

The viability of $L$. acidophilus before and after baking was demonstrated in Fig. 2. The results showed that, after baking, the different carriers provided the ability to protect L. acidophilus under the effect of temperature was different. The viability of $L$. acidophilus in the AMX and the AM samples was equivalent (P>0.05) of about 3.64 and $3.75 \mathrm{Log}$ (CFU/bread), corresponding to $66.5 \%$ and $63.2 \%, \mathrm{AX}$ and $\mathrm{A}$ had a greater loss of L. acidophilus during the baking, more than 4 Log (CFU/bread) (Fig. 2). The viability of L. acidophilus in the control sample had a significant loss, reaching $3.11 \mathrm{Log}$ (CFU/bread) compared to $11.45 \mathrm{Log}$ (CFU/bread) before baking, corresponding to the survival rate of only $32.90 \%$, this was much lower than microcapsules, proving microencapsulation help to protect L. acidophilus against high temperature.

A study by Zhang et al. (2014) on the viability of Bifidobacterium lactic $\mathrm{Bb} 12$, proving that probiotics exist during baking at $205^{\circ} \mathrm{C}$ for 12 minutes (Zhang et al., 2014). This proves the feasibility of developing probiotic bread lines. In addition, during baking, the temperature inside biscuits are lower than the baking temperature, which increases the probiotic surviving inside biscuits (Reid et al., 2007). The study of Lieu et al. (2017) showed that maltodextrin provided the ability to protect $L$. casei against the high-temperature influence during the spray-drying process (Lieu et al., 2017). In the present study, the presence of maltodextrin in AMX and AM improved the ability of $L$. acidophilus to protect against the high temperatures of the baking process (Fig. 2). This suggested that maltodextrin is necessary to protect probiotic against the high-temperature.

The viability of L. acidophilus during 8 days of storage at $4{ }^{\circ} \mathrm{C}$ was demonstrated in Fig 3 . The results showed that the viability of L. acidophilus in AX, AM and AMX slightly increased in the first two days of storage which were $0.76 ; 0.56 ; 0.75 \mathrm{Log}(\mathrm{CFU} / \mathrm{bread})$ respectively and then decreased in a small amount from day 2 to day 8 of the experiment. The control sample also recorded a slight increase from the first day was 3.11 Log (CFU/bread) and reach $4.02 \log$ (CFU/bread) after 8 days of storage. $L$. acidophilus concentration in carrier A decreased on the first day (6.33 $\mathrm{Log}(\mathrm{CFU} / \mathrm{bread})$ ) to the day 8 (5.8 $\mathrm{Log}$ (CFU/bread).

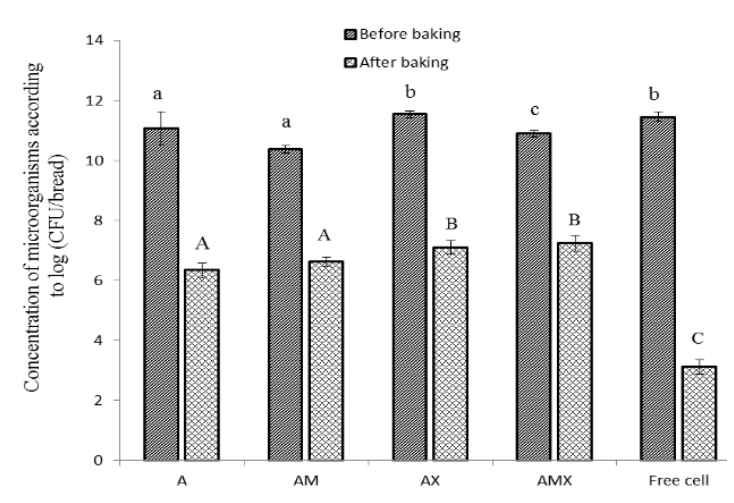

Fig 2. Lactobacillus acidophilus bacterial survival with high temperature (A: alginate 2\%; AM: alginate $2 \%+$ maltodextrin 1\%; AX: alginate $2 \%+$ xanthan gum $1 \%$; AMX: alginate $2 \%+$ maltodextrin $1 \%+$ xanthan gum $0,1 \%){ }^{\text {a-d. }}{ }^{A-C}$ represents a significant difference $(\mathrm{P}<0.05)$

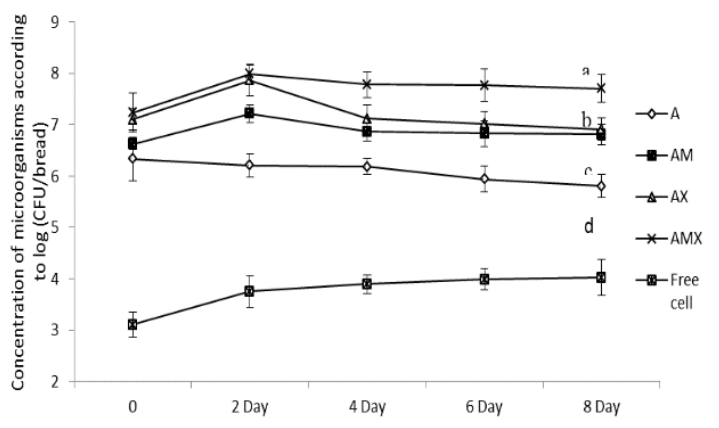

Fig 3. Survival of L. acidophilus during bread storage (A: alginate 2\%; AM: alginate 2\% + maltodextrin 1\%; AX: alginate $2 \%+$ xanthan gum $1 \%$; AMX: alginate $2 \%+$ maltodextrin $1 \%$ + xanthan gum $0.1 \%$ ). ${ }^{\text {a-d }}$ representing significant differences $(\mathrm{P}<0.05)$ in each experiment.

The slight increase in L. acidophilus viability during the first two days of storage due to L. acidophilus after baking using the substrate in the bread for further growth, which produced probiotic higher than dying probiotic. This phenomenon leads to the increasing the amount of $L$. acidophilus in the first 2 days of storage. From day 2 onwards, probiotic was decreased the viability which would due to the storage condition influence.

The influence of encapsulated particles on the probiotic viability during storage have been reported in the previous study. The study of Trabelsi et al. (2014) indicated that the protective effect of alginate in combination with polymer compounds on the L. plantarum viability showed better than using alginate alone during 35 days of storage at $4^{\circ} \mathrm{C}$ (Trabelsi et al., 2014). The similar result was observed in the present study, the viability of L. acidophilus in the alginate carrier that uses alone had the lowest compared to others (Fig 3). Compared to the preservation of probiotic bread and conventional bread, these two types have similar storage times. However, with the added benefit of probiotic 
for consumers, this product will have superiority over traditional bread products. In this study, different carriers will provide the ability to protect $L$. acidophilus bacteria under varying temperatures differently, both AM and AMX carriers providing the viability of L. acidophilus more than $80 \%$. Both contain maltodextrin in their constituents.

The Survived of L. acidophilus in Simulated Gastric Fluid (SGF) and Intestinal Fluid (SIF)

The L. acidophilus viability in the SGF and SIF condition was demonstrated in Fig 4. After 2 hours of incubation in SGF, the viability of L. acidophilus in AMX and AX was reduced respectively from 7.70 and $6.92 \mathrm{Log}$ (CFU/bread) to 3.42 and $2.57 \mathrm{Log}$ (CFU/bread) which corresponds to $44.4 \%$ and $37.20 \%$ respectively. The viability of L. acidophilus was not found in A, AM and the control samples (Fig 4). Continuing testing in the environment of SIF for 3 hours, the viability of $L$. acidophilus in the AMX sample was decreased to $2.25 \mathrm{log}$ (CFU/bread), whereas the L. acidophilus viability was not recorded in the AX sample.

Survival ability in SGF and SIF conditions is an important criterion for assessing the effectiveness of probiotics. The study by Fareez et al. (2015) states that the addition of xanthan to the carrier's constituents helped to improve the survival of $L$. plantarum LAB12 under SGF and SIF conditions. The interaction of xanthan gum and the acidic environment of gastric juices have explained the probiotic activity of xanthan gum in combination with alginate. The negative charge structure of Xanthan gum can bind to $\mathrm{H}^{+}$ions, reducing the effect of an acidic environment on the metabolism of bacteria (JiménezPranteda et al., 2012). In addition, maltodextrin would act as potential prebiotic to improve the viability of $L$. case $i$ in SGF condition (Đông et al., 2015). Chen et al. (2017) indicated that xanthan gum was added to the carrier matrix, which leads to the structure of the particles decreases their porosity, as the ion exchange between xanthan gum and simulated intestinal fluid can reduce the porosity of the microencapsulated matrix (Chen et al., 2017). In the present study, xanthan gum in the alginate matrix helped to improve the probiotic viability and was more improved in the present of maltodextrin (Fig. 4).

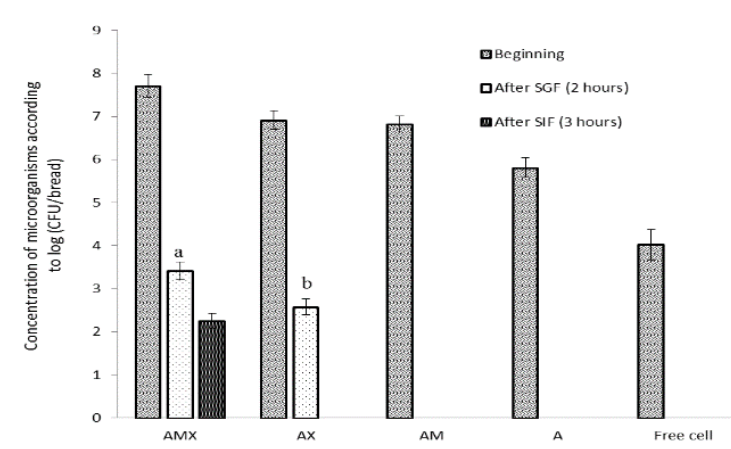

Fig 4. Survival of L. acidophilus in SGF and SIF after 8 days (A: alginate 2\%; AM: alginate $2 \%+$ maltodextrin $1 \%$; AX: alginate $2 \%+$ xanthan gum $1 \%$; AMX: alginate $2 \%$ + maltodextrin $1 \%$ + xanthan gum $0.1 \%$ ). a-b represents significant differences $(\mathrm{P}<0.05)$ in each experiment.
The results obtained suggest that, xanthan gum and maltodextrin is necessary to improve the probiotic viability. The combination of xanthan gum and maltodextrin in alginate matrix, eventually leading to have dual efficiency: First, xanthan gum would act as buffers that reduce acid activity; Second, maltodextrin act as potential prebiotic that improve the viability of probiotic

\section{Conclusion}

Application of probiotic micro-encapsulation technology in bread production is an economic research. Selecting the carrier has important meaning in increasing the survival rate of probiotic bacteria in processed, preserved and in the digestive environment. The results indicated that the addition of probiotics to bread was highly feasible. In the tested carrier agents, the best encapsulation yield was achieved with two carrier matrixes were AMX and AX (92.9\% and $92.37 \%)$, which contained xanthan gum in the carrier matrix. Maltodextrin acting as protective agent of $L$. acidophilus against high temperature, the viability of $L$. acidophilus was improved in the alginate matrix that contains maltodextrin compared to $\mathrm{AX}$ and $\mathrm{A}$ when baking at $185^{\circ} \mathrm{C}$ for 13 minutes. The combination of maltodextrin and xanthan gum in the alginate matrix provides the best survivability during storage (7.7 Log CFU/bread), as well as in simulated gastric fluid (SGF) and intestinal fluid (SIF) compared with the three systems carrier A, AM, AX. Xanthan gum shows its potential in protecting L. acidophilus in simulated gastric fluid (SGF) and intestinal fluid (SIF). Maltodextrin demonstrates the probiotic benefits of limiting temperature effects during processing. The combination of several carriers in microcapsules helps to take advantage of individual carriers to improve probiotic protection. Production of probiotic bread with AMX carrier gave the best protection against L. acidophilus compared to the other carriers

\section{References}

Agrawal R. 2005. Probiotics: an emerging food supplement with health benefits. Food Biotechnology., vol. 19, no. 3, pp. $227-$ 246. DOI: 10.1080/08905430500316474

Chen L, Yang T, Song Y, Shu G, Chen H. 2017. Effect of xanthan-chitosan-xanthan double layer encapsulation on survival of Bifidobacterium BB01 in simulated gastrointestinal conditions, bile salt solution and yogurt. LWT-Food Science and Technology., vol. 81, pp. 274-280. DOI: 10.1016/j.lwt.2017.04.005

Ding W, Shah N. 2007. Acid, bile, and heat tolerance of free and microencapsulated probiotic bacteria. Journal of food science., vol. 72, no. 9, pp. M446-M450. DOI: 10.1111/j.1750-3841.2007.00565.x

Fareez IM, Lim SM, Mishra RK, Ramasamy K. 2015. Chitosan coated alginate-xanthan gum bead enhanced $\mathrm{pH}$ and thermotolerance of Lactobacillus plantarum LAB12. International journal of biological macromolecules., vol. 72, pp. 1419-1428. DOI: 10.1016/j.ijbiomac.2014.10.054

Hatakka K, Savilahti E, Pönkä A, Meurman JH, Poussa T, Näse L, Saxelin M, Korpela R. 2001. Effect of long term consumption of probiotic milk on infections in children attending day care centres: double blind, randomised trial. Bmj., vol. 322, no. 7298, p. 1327, DOI: 10.1136/bmj.322.7298.1327 
Hill C, Guarner F, Reid G, Gibson GR, Merenstein DJ, Pot B, Morelli L, Canani RB, Flint HJ, Salminen S, Calder PC. 2014. Expert consensus document: The International Scientific Association for Probiotics and Prebiotics consensus statement on the scope and appropriate use of the term probiotic. Nature Reviews Gastroenterology and Hepatology, 11(8), p. 506. DOI: $10.1038 /$ nrgastro.2014.66

Homayouni A, Ehsani MR, Azizi A, Yarmand MS, Razavi H. 2007. Effect of lecithin and calcium chloride solution on the microencapsulation process yield of calcium alginate beads. p. 597-606.

Jiménez-Pranteda ML, Poncelet D, Náder-Macías ME, Arcos A, Aguilera M, Monteoliva-Sánchez M, Ramos-Cormenzana A. 2012. Stability of lactobacilli encapsulated in various microbial polymers. Journal of bioscience and bioengineering., vol. 113, no. 2, pp. 179-184. DOI: 10.1016/j.jbiosc.2011.10.010

Kailasapathy K, Chin J. 2000. Survival and therapeutic potential of probiotic organisms with reference to Lactobacillus acidophilus and Bifidobacterium spp. Immunology \& Cell Biology., vol. 78, no. 1: pp. 80-88. DOI: 10.1046/j.14401711.2000.00886.x
Lieu MD, Dang TKT, Nguyen TH. 2017. Viability of microencapsulated Lactobacillus casei in synbiotic mayonnaise. Food Research., pp. 234 - 239. DOI: 10.26656/fr.2017.6.103

Reid AA, Champagne CP, Gardner N, Fustier P, Vuillemard JC. 2007. Survival in Food Systems of Lactobacillus rhamnosus R011 Microentrapped in Whey Protein Gel Particles., Journal of Food Science, 72(1), M031-M037. DOI: 10.1111/j.17503841.2006.00222.x

Sultana K, Godward G, Reynolds N, Arumugaswamy R, Peiris P, Kailasapathy K. 2000. Encapsulation of probiotic bacteria with alginate-starch and evaluation of survival in simulated gastrointestinal conditions and in yoghurt. International journal of food microbiology., vol. 62, no. 1-2, pp. 47-55. DOI: 10.1016/S0168-1605(00)00380-9

Trabelsi I, Ayadi D, Bejar W, Bejar S, Chouayekh H, Salah RB. 2014. Effects of Lactobacillus plantarum immobilization in alginate coated with chitosan and gelatin on antibacterial activity. International journal of biological macromolecules., vol. 64, pp. 84-89. DOI: 10.1016/j.ijbiomac.2013.11.031

Zhang L, Huang S, Ananingsih VK, Zhou W, Chen XD. 2014. A study on Bifidobacterium lactis Bb12 viability in bread during baking., Journal of Food Engineering, 122, 33-37. DOI: 10.1016/j.jfoodeng.2013.08.029 